\title{
Validez y confiabilidad de la versión en español de la escala multidimensional de la UNESP-Botucatu para evaluar el dolor postoperatorio en gatos
}

\author{
Validation of the spanish version of the UNESP-Botucatu multidimensional composite \\ pain scale to assess postoperative pain in cats
}

\author{
JT Brondani ${ }^{a^{*}}$, SPL Luna ${ }^{a}$, N Crosignani ${ }^{b}$, JI Redondo ${ }^{c}$, MM Granados ${ }^{d}$, \\ H Bustamante ${ }^{\mathrm{e}}$, C Palacios ${ }^{\mathrm{f}}$, P Otero ${ }^{\mathrm{g}}$ \\ ${ }^{\text {a}}$ Facultad de Medicina Veterinaria y Zootecnia, Universidad Estadual Paulista, Botucatu, Brasil. \\ ${ }^{b}$ Facultad de Veterinaria, Universidad de la República, Montevideo, Uruguay. \\ ${ }^{\mathrm{c} F a c u l t a d}$ de Veterinaria, Universidad CEU Cardenal Herrera, Valencia, España. \\ ${ }^{\mathrm{d}}$ Facultad de Veterinaria, Universidad de Córdoba, Córdoba, España. \\ eFacultad de Ciencias Veterinarias, Universidad Austral de Chile, Valdivia, Chile.

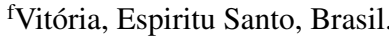 \\ gFacultad de Ciencias Veterinarias, Universidad de Buenos Aires, Buenos Aires, Argentina.
}

\begin{abstract}
SUMMARY
This study focused on the validation of the Spanish version of the UNESP-Botucatu multidimensional composite pain scale to assess postoperative pain in cats. The original scale in Portuguese was translated into Spanish by two independent translators, and summarised in one version by a third individual. The summarised version was back-translated and minor adjustments were made. The scale was reviewed by three anesthesiologists with Spanish as their first language, and the final version was submitted to psychometric testing. Thirty cats undergoing ovariohysterectomy were video recorded during the perioperative period. Six observers from different spanish speaking countries, who had Spanish as the first language watched the videos and determined the pain scores using the Spanish version of the scale, identifying the cats that should receive analgesics. Videos were reanalysed in a different order about 2 months after the first assessment. The sensitivity to change, content and construct validity were established by the significant change in pain scores in response to surgery and analgesics. The agreement between the evaluations of the 'gold standard' (researcher that developed the scale) and the other observers confirmed the criterion validity. Inter- and intra-rater reliability, evaluated by intra-class correlation coefficient, ranged from good to very good for all scale items. The cut-off point for rescue analgesia identified by Receiver Operating Characteristic curve was $>7$ with $92 \%$ of sensitivity and $91 \%$ of specificity. The Spanish version of the UNESP-Botucatu multidimensional composite pain scale is interpretable (has an optimal analgesic intervention score), valid and reliable instrument for assessing acute pain in cats undergoing ovariohysterectomy.
\end{abstract}

Key words: cats, pain, validation, scale.

\section{RESUMEN}

El objetivo de este estudio fue validar la versión en español de la escala multidimensional de la UNESP-Botucatu para evaluar el dolor postoperatorio en gatos. La escala original en portugués se tradujo al español y una versión sintetizada fue retrotraducida tras realizar pequeños ajustes. Tres anestesiólogos revisaron la escala (español de lengua materna), y la versión final se sometió a test psicométricos. Treinta gatas sometidas a ovariohisterectomía fueron filmadas durante el periquirúrgico. Seis observadores de diferentes culturas y con el español como lengua materna evaluaron los videos determinando las puntuaciones de dolor utilizando la escala en español, identificando a los animales que debían recibir analgesia adicional. Los videos se reevaluaron aproximadamente dos meses después del primer análisis. La sensibilidad al cambio, validez del contenido y constructo del instrumento se establecieron gracias a las alteraciones significativas en las puntuaciones de dolor en respuesta a la cirugía y analgesia. La concordancia entre las evaluaciones del patrón de referencia (investigador que desarrolló la escala) con los demás observadores confirmó la validez de criterio. La confiabilidad inter e intraobservadores evaluada mediante el coeficiente de correlación intraclase varió entre buena y muy buena en todos los ítems de la escala. La puntuación para intervención analgésica, identificada por la curva Receiver Operating Characteristic, fue de $>7$ con una sensibilidad del $92 \%$ y una especificidad del $91 \%$. La versión en español de la escala multidimensional de la UNESP-Botucatu es un instrumento interpretable (presenta una puntuación para intervención analgésica), válido y confiable para evaluar el dolor agudo en gatas sometidas a ovariohisterectomía.

Palabras clave: gatos, dolor, validación, escalas.

Aceptado: 08.05.2014

* jtbrondani@yahoo.com 


\section{INTRODUCCIÓN}

El auge actual de la medicina basada en la evidencia, resalta la importancia de estandarizar los métodos de evaluación del dolor en los pequeños animales para poder comparar los resultados de estudios diferentes. Para ello necesitamos escalas de evaluación del dolor que estén validadas en múltiples lenguas y culturas.

Los instrumentos unidimensionales, como las escalas analógica, visual, numérica y descriptiva simple, son universales y se utilizan ampliamente en la evaluación del dolor en los pequeños animales (Anil y col 2002). Sin embargo, el carácter extremadamente subjetivo de estas escalas provocan que sean inconsistentes y que haya variaciones en las observaciones cuando las utilizan observadores diferentes (Holton y col 1998). Para limitar la subjetividad y aumentar la precisión de la evaluación se han desarrollado escalas de evaluación del dolor específicas para una especie determinada (Holton y col 2001). Recientemente se ha desarrollado y validado un instrumento destinado a la evaluación del dolor postoperatorio en gatos, primero en portugués brasileño (Brondani y col 2011, 2012, 2013 ${ }^{\mathrm{a}}$ ), y posteriormente en inglés (Brondani y col 2013 ${ }^{\mathrm{b}}$ ).

La validación de una escala de evaluación del dolor en una lengua o cultura requiere un proceso riguroso y minucioso de traducción, adaptación cultural y evaluación de las propiedades psicométricas en ese idioma o cultura (Guillemin y col 1993, Beaton y col 2000). Las pruebas psicométricas evalúan la validez, confiabilidad y sensibilidad al cambio del instrumento. La validez es la capacidad de la escala para medir lo que ella se propone a medir (Morton y col 2005), y aunque haya sido tradicionalmente separada en tres aspectos, a saber, contenido, criterio y constructo, el enfoque principal debe ser la metodología de test de hipótesis (Streiner y Norman 2008).

La validez de contenido se refiere al grado en que un instrumento refleja un dominio específico de contenido de lo que se mide. Es el grado en que la medición representa al concepto medido (Streiner y Norman 2008). La validez de constructo determina la relación del instrumento con la teoría y la conceptualización teórica. La validez de constructo también se puede comprobar mediante el análisis factorial, que distingue las dimensiones subyacentes que establecen las relaciones entre los ítems del instrumento. Validez de criterio establece la eficacia de un instrumento de medición comparándola con algún criterio externo (Souza y Silva 2005).

La confiabilidad evalúa si el instrumento está midiendo algo de una forma reproducible. Puede ser determinada por la consistencia interna, que verifica la interrelación entre los diferentes ítems de la escala, o examinando la reproducibilidad de una medida cuando la evalúan diferentes observadores (confiabilidad interobservadores), o cuando se realiza en diferentes ocasiones (confiabilidad intraobservadores) (Streiner y Norman 2008). La sensibilidad al cambio evalúa la habilidad de la escala para detectar una alteración significativa en las puntuaciones de dolor en una dirección esperada, en respuesta a eventos que reducen el dolor, como analgésicos, o que producen dolor, como los procedimientos quirúrgicos (Baeyer y Spagrud 2007).

El español es la segunda lengua más difundida en el mundo. Por tanto, el objetivo de este estudio es validar la versión en español de la escala multidimensional de la Universidad UNESP-Botucatu para evaluar el dolor postoperatorio en gatos, siguiendo las directrices internacionales propuestas en la bibliografía (Beaton y col 2000, Streiner y Norman 2008, Souza y Rojjanasrirat 2011). La adaptación cultural incluyó la traducción, retrotraducción y análisis del contenido de la escala realizados por un comité de especialistas. En consecuencia, fue determinada la dimensionalidad, consistencia interna, sensibilidad al cambio, validez del contenido, constructo y criterio, confiabilidad inter e intraobservadores y la puntuación mínima para intervención analgésica. La hipótesis propuesta es que si se alcanza una adecuada traducción/adaptación cultural, la versión en español presentará propiedades psicométricas excelentes, como ya se observó en la escala original en portugués y en su versión en inglés.

\section{MATERIAL Y MÉTODOS}

\section{TRADUCCIÓN, RETROTRADUCCIÓN Y VALIDEZ DEL CONTENIDO}

Inicialmente la escala fue traducida del idioma original, el portugués brasileño, a la lengua española, por dos traductores independientes bilingües y cuya lengua materna era el español. Tras analizar las dos traducciones, una tercera persona que también tenía el español como primer idioma realizó una versión sintetizada. Posteriormente la escala en español (versión sintetizada) fue traducida de nuevo a la lengua portuguesa (traducción reversa o retrotraducción) por un traductor bilingüe diferente, que tenía el portugués como primer idioma. Este investigador no tuvo acceso a la escala original en portugués. A continuación, la escala en portugués originada por la traducción reversa se comparó con la escala original, y se hicieron pequeños ajustes en la escala en español para conservar la mayor equivalencia semántica.

Finalmente, tres anestesiólogos con conocimientos en el área de dolor y que tenían el español como lengua materna realizaron un análisis crítico detallado del contenido de la escala centrado en los matices de la adaptación cultural. Teniendo en cuenta las diferencias culturales entre los países que tienen el español como idioma oficial, fueron seleccionados dos investigadores de América Latina (Argentina y Chile) y otro de España. Después de modificar el contenido atendiendo a las sugerencias de los especialistas, se generó la versión final de la escala en español, que se estudió en los test psicométricos. 
PRUEBAS DE VALIDACIÓN DE LA ESCALA EN LA VERSIÓN EN ESPAÑOL A PARTIR DEL ANÁLISIS DE VIDEOS

Las propiedades psicométricas de la versión en español de la escala se estudiaron a partir del análisis de videos grabados durante el período perioperatorio de 30 gatas sometidas a una ovariohisterectomía (aprobación por la comisión de ética en experimentación animal de la FMVZUNESP-Botucatu con el protocolo No 20/2008). Para realizar el procedimiento quirúrgico se indujo la anestesia con propofol $(8 \mathrm{mg} / \mathrm{kg})$ IV y se mantuvo con isofluorano (1,5-2,0 CAM) en $100 \%$ de oxígeno, administrados por medio de un sistema sin reinhalación. Se utilizó fentanilo $(0,002 \mathrm{mg} / \mathrm{kg})$ IV como analgésico durante la cirugía. Las gatas fueron filmadas durante cinco minutos en cuatro momentos predeterminados del período perioperatorio: M1 (preoperatorio: de 18 a 24 horas antes de la cirugía), M2 (postoperatorio, antes del uso de analgesia de rescate: de 30 minutos a 1 hora tras la cirugía), M3 (postoperatorio, tras administrar la analgesia de rescate: 4 horas tras administrar conjuntamente los siguientes analgésicos: morfina $0,2 \mathrm{mg} / \mathrm{kg}$ IM, ketoprofeno $2 \mathrm{mg} / \mathrm{kg} \mathrm{SC}$ y dipirona $25 \mathrm{mg} /$ kg IV), M4 (postoperatorio, 24 horas después de acabar la cirugía). Las gatas recibieron la asociación de analgésicos anteriormente citada al acabar la filmación en el M2.

Para realizar las pruebas de validez y confiabilidad seis anestesiólogos que tenían el español como primera lengua, oriundos de Argentina (1), Chile (1), España (3) y Uruguay (1), analizaron los videos y determinaron las puntuaciones de dolor utilizando la versión en español de la escala multidimensional de la UNESP-Botucatu (cuadro 1). Para que los observadores fuesen ciegos, es decir, para que no supieran los diferentes momentos de evaluación, durante la organización de los discos de almacenamiento de datos se alteró el orden cronológico de los videos y durante el período preoperatorio se hizo una cura en el lugar en el que estaría la herida quirúrgica.

Dimensionalidad y consistencia interna. Para confirmar la estructura multidimensional de la escala original la versión en español fue sometida a un análisis factorial exploratorio a partir de la técnica de análisis de los componentes principales, con rotación varimax en la matriz de correlación. El método de rotación varimax busca maximizar la varianza en los factores, lo que permite una mejor representación de las variables (Kaiser 1958). La identificación de los factores se basó en el criterio Kaiser, que sugiere la permanencia de todos los factores con autovalor mayor a la unidad (Kaiser 1958). La estructura factorial se determinó considerando ítems con carga factorial y comunalidad mayor que 0,5. La consistencia interna se estableció como un todo para la escala y para cada una de las subescalas identificadas en el análisis factorial.

Sensibilidad al cambio y validez del criterio, contenido y constructo. La validez del criterio fue establecida a partir de la concordancia entre las puntuaciones de dolor registradas por los observadores "ciegos" con el español como idioma materno, y las puntuaciones de dolor registradas por el investigador brasileño que desarrolló la escala (patrón de referencia).

La validez del contenido se basó en la premisa de que si la escala mide dolor, los animales en el período preoperatorio de la cirugía electiva (ovariohisterectomía) deberían presentar puntuaciones de dolor significativamente menores al compararlos con el período postoperatorio y tras haber sido sometidos a una situación dolorosa. De esta forma se evaluó si las puntuaciones de dolor en el M2 aumentaron de modo significativo comparados con el M1.

La validez del constructo se determinó al considerarse que si los analgésicos reducen el dolor, las puntuaciones deberían disminuir después de la administración de analgésicos. De esta forma, las puntuaciones de dolor en el M3 deberían ser menores que en el M2. También se evaluó la validez del constructo a partir de las alteraciones en las puntuaciones de dolor durante el período postoperatorio, ya que el dolor agudo es de duración limitada y tiende a disminuir. Para ello se compararon las puntuaciones de dolor en el M2 con las obtenidas en el M4. Este método también se utilizó para evaluar su sensibilidad al cambio.

Confiabilidad inter e intraobservadores. La confiabilidad inter e intraobservadores se evaluó en cada uno de los ítems de la escala. Es decir, se determinó el grado de concordancia entre los diferentes observadores con el español como idioma nativo y el grado de concordancia entre las evaluaciones hechas por el mismo observador en diferentes ocasiones, respectivamente. Para la determinación de la confiabilidad intraobservadores se entregaron nuevos DVD, reorganizando el orden de los animales y los momentos de evaluación. El tiempo transcurrido entre el primer y el segundo análisis fue de uno a dos meses.

Definición de la puntuación del rescate analgésico. Para identificar la puntuación mínima de la escala relacionada con la necesidad de una intervención analgésica, los observadores "ciegos", al terminar el análisis de cada video, indicaron la necesidad o no de administrar analgésicos, según su experiencia clínica. Para ello, en el formulario de evaluación se incluyó la siguiente pregunta: de acuerdo con su experiencia clínica, ¿cree usted que es necesario administrar analgesia adicional?

La puntuación del rescate analgésico se identificó mediante el análisis de la Curva ROC (Receiver Operating Characteristic), en la que se sugirieron diferentes puntos de corte. El punto óptimo fue representado por el valor en el que la sensibilidad y especificidad eran mayores simultáneamente. También se calculó el área bajo la curva, que indica la capacidad discriminativa del método, esto es, que evalúa la exactitud de la habilidad del instrumento para clasificar correctamente a los individuos con y sin dolor. 
Cuadro 1. Escala multidimensional de la UNESP-Botucatu para la evaluación del dolor postoperatorio en gatos.

UNESP-Botucatu multidimensional composite pain scale to assess postoperative pain in cats.

\begin{tabular}{|c|c|c|}
\hline \multicolumn{3}{|c|}{ Subescala 1: Alteración psicomotriz (0-15) } \\
\hline $\begin{array}{l}\stackrel{\pi}{\Xi} \\
\stackrel{\tilde{E}}{0} \\
\stackrel{0}{0}\end{array}$ & $\begin{array}{l}\text { - El gato adopta una postura considerada normal para la especie y parece relajado (se mueve con normalidad). } \\
\text { - El gato adopta una postura considerada normal para la especie pero parece tenso (se mueve poco o reticente a } \\
\text { moverse). } \\
\text { - El gato está sentado o en decúbito esternal, con la columna arqueada y la cabeza baja, o el gato está en decúbito } \\
\text { lateral con los miembros pelvianos extendidos o recogidos. } \\
\text { - El gato cambia frecuentemente de posición intentando encontrar una postura cómoda. }\end{array}$ & $\begin{array}{l}0 \\
1\end{array}$ \\
\hline 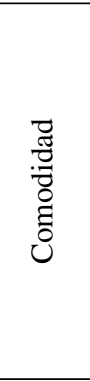 & $\begin{array}{l}\text { - El gato está cómodo, despierto o adormecido, y se muestra receptivo cuando se estimula (interactúa con el } \\
\text { observador o se interesa por el ambiente). } \\
\text { - El gato está quieto y se muestra poco receptivo cuando se estimula (interactúa poco con el observador o no se } \\
\text { interesa mucho por el ambiente). } \\
\text { - El gato está quieto y desconectado del ambiente (no interactúa con el observador aunque se lo estimule o no se } \\
\text { interesa por el ambiente). El gato puede dar la espalda al observador (mirando hacia el fondo de la jaula). } \\
\text { - El gato está incómodo, se muestra inquieto (cambia frecuentemente de posición) y está desconectado del } \\
\text { ambiente o está poco receptivo cuando es estimulado. El gato puede dar la espalda al observador (mirando hacia } \\
\text { el fondo de la jaula). }\end{array}$ & $\begin{array}{l}0 \\
1 \\
2 \\
3\end{array}$ \\
\hline 宽 & $\begin{array}{l}\text { - El gato se mueve normalmente (se mueve rápidamente cuando se abre la jaula; fuera de la jaula se mueve de } \\
\text { forma espontánea después de ser estimulado o manipulado). } \\
\text { - El gato se mueve más de lo normal (dentro de la jaula se mueve constantemente de un lado a otro). } \\
\text { - El gato está más quieto de lo normal (puede dudar en salir de la jaula y si se saca intenta volver a entrar; fuera } \\
\text { de la jaula se mueve poco después de ser estimulado o manipulado). } \\
\text { - El gato se resiste a moverse (puede dudar en salir de la jaula y si se saca intenta volver a entrar; fuera de la jaula } \\
\text { no se mueve, incluso después de ser estimulado o manipulado). }\end{array}$ & $\begin{array}{l}0 \\
1 \\
2 \\
3\end{array}$ \\
\hline 总 & $\begin{array}{l}\text { Observe y escoja las opciones que describan mejor el estado mental del gato: } \\
\text { A - Contento: El gato está alerta e interesado por el ambiente (explora los alrededores); es amigable e interactúa } \\
\text { con el observador (juega o responde a estímulos). } \\
\text { * Inicialmente el gato puede jugar con el observador para distraerse del dolor. Observe con atención para } \\
\text { diferenciar "intento de distracción" de "juegos de satisfacción". } \\
\text { B - Desinteresado: El gato no interactúa con el observador (no está interesado en jugar o juega poco; no } \\
\text { responde a las caricias y llamadas del observador)*. En gatos a los que no les guste jugar, evalúe la } \\
\text { interacción con el observador mediante la respuesta a las caricias y llamadas. } \\
\text { C - Indiferente: El gato no está interesado en el ambiente (no tiene curiosidad, no explora los alrededores). * } \\
\text { Inicialmente el gato puede estar receloso y no querer explorar los alrededores. El observador debe manipular } \\
\text { al gato (sacarlo de la jaula o alterar su postura) y animarlo a que se mueva. } \\
\text { D - Ansioso: El gato está asustado (intenta esconderse o escapar) o nervioso (está impaciente y gruñe o bufa } \\
\text { cuando se le acaricia o manipula). } \\
\text { E - Agresivo: El gato está agresivo (intenta morder o arañar cuando se le acaricia o manipula). } \\
\text { - Presencia de estado mental A. } \\
\text { - Presencia de uno de los estados mentales B, C, D o E. } \\
\text { - Presencia de dos de los estados mentales B, C, D o E. } \\
\text { - Presencia de tres o de todos los estados mentales B, C, D o E. }\end{array}$ & $\begin{array}{l}\text { D } \\
\text { E } \\
0 \\
1 \\
2 \\
3\end{array}$ \\
\hline 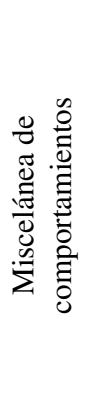 & $\begin{array}{l}\text { Observe y escoja las opciones que describan mejor el comportamiento del gato: } \\
\text { A - El gato está acostado y quieto, sin embargo mueve la cola. } \\
\text { B - El gato recoge y extiende los miembros pelvianos o contrae los músculos abdominales (flanco). } \\
\text { C - El gato está con los ojos parcialmente cerrados (semicerrados). } \\
\text { D - El gato se lame o muerde la herida quirúrgica. } \\
\text { - Todos los comportamientos descritos están ausentes. } \\
\text { - Presencia de uno de los comportamientos descritos. } \\
\text { - Presencia de dos de los comportamientos descritos. } \\
\text { - Presencia de tres o de todos los comportamientos descritos. }\end{array}$ & $\begin{array}{l}\text { A } \\
\text { B } \\
\text { C } \\
\text { D } \\
0 \\
1 \\
2 \\
3\end{array}$ \\
\hline
\end{tabular}


(continuación cuadro 1)

\begin{tabular}{|c|c|c|}
\hline 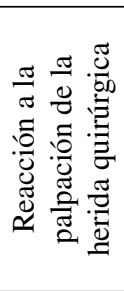 & $\begin{array}{l}\text { - El gato no reacciona cuando se toca y se presiona la herida quirúrgica; o no muestra una respuesta diferente a } \\
\text { la reacción preoperatoria (si se llevó a cabo una evaluación basal). } \\
\text { - El gato no reacciona cuando se toca la herida quirúrgica, pero sí cuando se presiona, pudiendo vocalizar o } \\
\text { intentar morder. } \\
\text { - El gato reacciona cuando se toca y se presiona la herida quirúrgica, pudiendo vocalizar o intentar morder. } \\
\text { - El gato reacciona cuando el observador se aproxima a la herida quirúrgica, pudiendo vocalizar o intentar } \\
\text { morder. No permite la palpación de la herida quirúrgica. }\end{array}$ & $\begin{array}{l}2 \\
3\end{array}$ \\
\hline 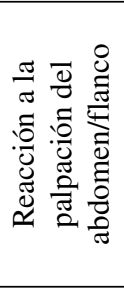 & $\begin{array}{l}\text { - El gato no reacciona cuando se toca y se presiona el abdomen/flanco; o no muestra una respuesta diferente a la } \\
\text { reacción preoperatoria (si se llevó a cabo una evaluación basal). El abdomen/flanco no está tenso. } \\
\text { - El gato no reacciona cuando se toca el abdomen/flanco, pero sí cuando se presiona. El abdomen/flanco está } \\
\text { tenso. } \\
\text { - El gato reacciona cuando se toca y se presiona el abdomen/flanco. El abdomen/flanco está tenso. } \\
\text { - El gato reacciona cuando el observador se aproxima al abdomen/flanco, pudiendo vocalizar o intentar morder. } \\
\text { No permite la palpación del abdomen/flanco. }\end{array}$ & $\begin{array}{l}0 \\
1 \\
2 \\
3\end{array}$ \\
\hline 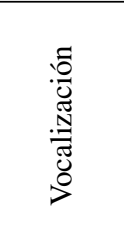 & $\begin{array}{l}\text { - El gato está en silencio o ronronea cuando es estimulado o maúlla interactuando con el observador, pero no } \\
\text { gruñe ni bufa. } \\
\text { - El gato ronronea espontáneamente (sin ser estimulado o manipulado por el observador). } \\
\text { - El gato gruñe o bufa cuando es manipulado por el observador (cuando el observador altera la posición corporal } \\
\text { del gato). } \\
\text { - El gato gruñe o bufa espontáneamente (sin ser estimulado o manipulado por el observador). }\end{array}$ & $\begin{array}{l}0 \\
1 \\
2 \\
3\end{array}$ \\
\hline \multicolumn{3}{|c|}{$\begin{array}{ll} & \text { Subescala 3: variables fisiológicas }(0-6)\end{array}$} \\
\hline 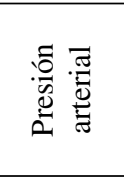 & $\begin{array}{l}\text { - } 0 \% \text { a } 15 \% \text { superior al valor preoperatorio. } \\
\text { - } 16 \% \text { a } 29 \% \text { superior al valor preoperatorio. } \\
\text { - } 30 \% \text { a } 45 \% \text { superior al valor preoperatorio. } \\
\text { - }>45 \% \text { superior al valor preoperatorio. }\end{array}$ & $\begin{array}{l}0 \\
1 \\
2 \\
3\end{array}$ \\
\hline $\begin{array}{l}\stackrel{0}{0} \\
\stackrel{0}{0} \\
\stackrel{2}{4}\end{array}$ & $\begin{array}{l}\text { - El gato come normalmente. } \\
\text { - El gato come más de lo normal. } \\
\text { - El gato come menos de lo normal. } \\
\text { - El gato gruñe o bufa espontáneamente (sin ser estimulado o manipulado por el observador). }\end{array}$ & $\begin{array}{l}0 \\
1 \\
2 \\
3\end{array}$ \\
\hline \multicolumn{3}{|c|}{ Resultado total (0-30) } \\
\hline $\begin{array}{l}\text { Inicialme } \\
\text { o movién } \\
\text { (véase el } \\
\text { Abra la p } \\
\text { reacción: } \\
\text { gusta ser } \\
\text { (llámelo } \\
\text { el gato se } \\
\text { Para final } \\
\text { al tocar s } \\
\text { presión d } \\
\text { quirúrgic } \\
\text { *Para la } \\
\text { comida h } \\
\text { independ } \\
\text { animal. }\end{array}$ & $\begin{array}{l}\text { te, observe el comportamiento del gato sin abrir la jaula. Verifique si el gato está descansando (en decúbito o ser } \\
\text { lose, interesado o no en el ambiente, en silencio o vocalizando. Examine la presencia de comportamientos espec } \\
\text { partado de "Comportamiento"). } \\
\text { erta de la jaula y observe si el animal intenta salir rápidamente o si duda en intentar salir. Aproxímese al gato y eva } \\
\text { está amigable, agresivo, asustado, indiferente o vocaliza. Toque al gato e interactúe con él, observe si está recepti } \\
\text { cariciado o demuestra interés por jugar). Si el gato duda en intentar salir de la jaula, incentívelo a moverse estimulá } \\
\text { or el nombre y acarícielo) y manipulándolo (altere su posición corporal o sáquelo de la jaula). Observe si fuera de la } \\
\text { nueve espontáneamente, de forma tímida o se niega a moverse. Ofrézcale un alimento palatable y observe su respu } \\
\text { zar, coloque con cuidado el gato en decúbito lateral o esternal y tome la presión arterial. Observe la reacción del a } \\
\text { avemente el abdomen/flanco (deslice suavemente los dedos sobre el área) y después presione con cuidado (apliqu } \\
\text { recta sobre el área con los dedos). Espere unos minutos y repita la misma secuencia de movimientos sobre la } 1 \\
\text { para evaluar la reacción ante este estímulo. } \\
\text { valuación del apetito en el postoperatorio inmediato ofrezca una pequeña cantidad de alimento palatable (por eje } \\
\text { meda enlatada) después de la recuperación anestésica. En este momento, la mayoría de los gatos comerá normaln } \\
\text { entemente de la presencia o ausencia de dolor. Pasado un tiempo, ofrezca de nuevo la comida y observe la reacci }\end{array}$ & \\
\hline
\end{tabular}

\section{ANÁLISIS ESTADÍSTICO}

La consistencia interna de la escala en español se evaluó a partir del cálculo del coeficiente alfa de Cronbach, considerando valores aceptables mayores de 0,7 (Jensen 2003). La concordancia entre los observadores "ciegos" con el español como lengua materna y el patrón de referencia, se evaluó en cada ítem de la escala mediante el coeficiente de confiabilidad Kappa ponderado con $95 \%$ de intervalo de confianza, teniendo en cuenta los cuatro momentos de evaluación agrupados. La validez del contenido y del constructo de las alteraciones en las puntuaciones de dolor se evaluaron con la prueba de rangos con signo de Wilcoxon, con un nivel de significancia del $5 \%$. 
La confiabilidad intra e interobservadores fue verificada mediante el coeficiente de correlación intraclase con $95 \%$ de intervalo de confianza. Se utilizó el modelo de dos factores con concordancia absoluta -los observadores son los mismos para todos los individuos; todos los individuos son evaluados por los mismos observadores y las diferencias sistemáticas son importantes. La concordancia evaluada mediante el coeficiente de confiabilidad Kappa ponderado y mediante el coeficiente de correlación intraclase se interpretó mediante la clasificación de Altman (1991): 0,81-1,0 muy buena; 0,61-0,80 buena; $0,41-0,6$ moderada; $0,21-0,4$ razonable; $<0,2$ pobre. Los análisis estadísticos se realizaron con los programas SPSS ${ }^{\circledR}$ versión 12.0.1 (SPSS Inc, Illinois, Estados Unidos) y MedCalc® versión 12.4.0 (MedCalc Software bvba, Ostend, Bélgica).

\section{RESULTADOS}

\section{DIMENSIONALIDAD Y CONSISTENCIA INTERNA}

La versión en español de la escala presentó tres factores con autovalores de magnitud 3,88; 2,57 y 1,45, y con ítems con carga factorial y comunalidad satisfactorias $(>0,5)$. Las dimensiones o subescalas identificadas se denominaron (1) "Alteración Psicomotriz", (2) "Protección del área dolorida y expresión vocal del dolor" y (3) "Variables Fisiológicas" y contribuyeron con 38,8\%, 25,7\% y $14,5 \%$ de la variancia total, respectivamente. La subescala 1 está compuesta por los ítems: postura, comodidad, actividad, actitud y miscelánea de comportamientos. La subescala 2 representa los ítems reacción a la palpación de la herida quirúrgica, reacción a la palpación del abdomen/flanco y vocalización; y la subescala 3 engloba la presión arterial y el apetito. El coeficiente alfa de Cronbach como un todo para la escala y las subescalas 1 y 2 fue de 0,$92 ; 0,94$ y 0,85 , respectivamente, lo que indica una excelente consistencia interna. La subescala 3 presentó una consistencia interna moderada de 0,55 .

\section{SENSIBILIDAD AL CAMBIO Y VALIDEZ DE CRITERIO, CONTENIDO Y CONSTRUCTO}

Considerando los cuatro momentos de evaluación, la concordancia entre el "patrón de referencia" y los diferentes observadores "ciegos" con el español como primera lengua fue muy buena en los ítems "reacción a la palpación de la herida quirúrgica" y "reacción a la palpación del abdomen/ flanco". En los demás ítems la concordancia varió entre buena y muy buena (cuadro 2). Tras confirmar la multidimensionalidad de la escala en la versión en español se realizaron los test de validez de contenido y constructo

Cuadro 2. Concordancia entre el evaluador "patrón de referencia" y los diferentes observadores "ciegos" con el español como lengua materna, evaluada por el coeficiente de confiabilidad kappa ponderado ( $95 \%$ de intervalo de confianza), para cada ítem de la escala, incluyendo todos los momentos de evaluación (preoperatorio, postoperatorio antes y luego de la analgesia de rescate y 24 horas posterior al término de la cirugía).

Agreement between blinded observers with Spanish as the first language and 'gold-standard', assessed by weight kappa coefficient (95\% of confidence interval), for each scale item considering all assessment times (preoperative, postoperative before and after rescue analgesia, and 24 hours after the end of surgery).

\begin{tabular}{|c|c|c|c|c|c|c|}
\hline \multirow{2}{*}{ Ítems de la escala } & \multicolumn{6}{|c|}{$\begin{array}{c}\text { Observadores "ciegos” con español como lengua materna } \\
\text { Anestesiólogo }\end{array}$} \\
\hline & 1 & 2 & 3 & 4 & 5 & 6 \\
\hline Postura & $\begin{array}{c}0,83 \\
(0,77-0,89)\end{array}$ & $\begin{array}{c}0,78 \\
(0,72-0,85)\end{array}$ & $\begin{array}{c}0,77 \\
(0,67-0,86)\end{array}$ & $\begin{array}{c}0,86 \\
(0,77-0,95)\end{array}$ & $\begin{array}{c}0,90 \\
(0,86-0,95)\end{array}$ & $\begin{array}{c}0,93 \\
(0,90-0,97)\end{array}$ \\
\hline Comodidad & $\begin{array}{c}0,80 \\
(0,73-0,87)\end{array}$ & $\begin{array}{c}0,82 \\
(0,74-0,90)\end{array}$ & $\begin{array}{c}0,67 \\
(0,54-0,79)\end{array}$ & $\begin{array}{c}0,90 \\
(0,85-0,95)\end{array}$ & $\begin{array}{c}0,88 \\
(0,83-0,94)\end{array}$ & $\begin{array}{c}0,83 \\
(0,75-0,91)\end{array}$ \\
\hline Actividad & $\begin{array}{c}0,75 \\
(0,66-0,84)\end{array}$ & $\begin{array}{c}0,77 \\
(0,68-0,86)\end{array}$ & $\begin{array}{c}0,78 \\
(0,69-0,86)\end{array}$ & $\begin{array}{c}0,85 \\
(0,77-0,92)\end{array}$ & $\begin{array}{c}0,83 \\
(0,75-0,91)\end{array}$ & $\begin{array}{c}0,81 \\
(0,72-0,90)\end{array}$ \\
\hline Actitud & $\begin{array}{c}0,75 \\
(0,67-0,83)\end{array}$ & $\begin{array}{c}0,82 \\
(0,75-0,90)\end{array}$ & $\begin{array}{c}0,76 \\
(0,65-0,87)\end{array}$ & $\begin{array}{c}0,85 \\
(0,78-0,91)\end{array}$ & $\begin{array}{c}0,85 \\
(0,79-0,91)\end{array}$ & $\begin{array}{c}0,86 \\
(0,80-0,92)\end{array}$ \\
\hline $\begin{array}{l}\text { Miscelánea de } \\
\text { comportamientos }\end{array}$ & $\begin{array}{c}0,91 \\
(0,86-0,96)\end{array}$ & $\begin{array}{c}0,75 \\
(0,67-0,84)\end{array}$ & $\begin{array}{c}0,74 \\
(0,63-0,85)\end{array}$ & $\begin{array}{c}0,94 \\
(0,91-0,96)\end{array}$ & $\begin{array}{c}0,93 \\
(0,89-0,96)\end{array}$ & $\begin{array}{c}0,95 \\
(0,92-0,98)\end{array}$ \\
\hline $\begin{array}{l}\text { Palpación del abdomen/ } \\
\text { flanco }\end{array}$ & $\begin{array}{c}0,91 \\
(0,87-0,94)\end{array}$ & $\begin{array}{c}0,89 \\
(0,84-0,93)\end{array}$ & $\begin{array}{c}0,85 \\
(0,79-0,91)\end{array}$ & $\begin{array}{c}0,88 \\
(0,82-0,95)\end{array}$ & $\begin{array}{c}0,84 \\
(0,76-0,92)\end{array}$ & $\begin{array}{c}0,84 \\
(0,77-0,91)\end{array}$ \\
\hline Apetito & $\begin{array}{c}0,86 \\
(0,78-0,95)\end{array}$ & $\begin{array}{c}0,90 \\
(0,81-0,98)\end{array}$ & $\begin{array}{c}0,67 \\
(0,49-0,85)\end{array}$ & $\begin{array}{c}0,75 \\
(0,57-0,94)\end{array}$ & $\begin{array}{c}0,87 \\
(0,74-1,00)\end{array}$ & $\begin{array}{c}0,94 \\
(0,88-0,99)\end{array}$ \\
\hline Vocalización & $\begin{array}{c}0,91 \\
(0,83-0,98)\end{array}$ & $\begin{array}{c}0,80 \\
(0,67-0,92)\end{array}$ & $\begin{array}{c}0,75 \\
(0,62-0,87)\end{array}$ & $\begin{array}{c}0,76 \\
(0,60-0,91)\end{array}$ & $\begin{array}{c}0,72 \\
(0,60-0,85)\end{array}$ & $\begin{array}{c}0,87 \\
(0,79-0,95)\end{array}$ \\
\hline
\end{tabular}

0,81-1,0 concordancia muy buena; $0,61-0,80$ buena; $0,41-0,60$ moderada; $0,21-0,4$ razonable; $<0,2$ pobre. 
para la puntuación total de la escala y para cada una de las subescalas identificadas en el análisis factorial. Al considerarse la evaluación de los seis anestesiólogos que tenían el español como lengua materna, la puntuación total de la escala y de las tres subescalas aumentó significativamente tras el procedimiento quirúrgico, y disminuyó de forma elocuente en el postoperatorio después de administrar la analgesia de rescate y 24 horas después de la cirugía (cuadro 3).

\section{CONFIABILIDAD INTER E INTRAOBSERVADORES}

La concordancia entre los observadores "ciegos" con el español como primer idioma varió entre buena y muy buena en todos los ítems de la escala: postura $0,76(0,69-0,82)$, comodidad $0,75(0,68-0,81)$, actividad $0,73(0,67-0,79)$, actitud $0,73(0,65-0,80)$, miscelánea de comportamiento $0,80(0,75-0,85)$, reacción a la palpación de la herida quirúrgica $0,85(0,81-0,89)$, reacción a la palpación del abdomen/flanco $0,84(0,80-0,87)$, apetito $0,72(0,66-0,78)$ y vocalización $0,75(0,70-0,80)$. La concordancia intraobservadores varió entre buena y muy buena en todos los ítems de la escala, excepto en los ítems reacción a la palpación de la herida quirúrgica y reacción a la palpación del abdomen/flanco, que presentaron confiabilidad muy buena para todos los evaluadores.

\section{DEFINICIÓN DE LA PUNTUACIÓN DEL RESCATE ANALGÉSICO}

El punto de corte óptimo se estimó en $>7$, con una sensibilidad del 91,9\% (95\% IC: $86,8-95,5 \%)$, y una especificidad del 91,2\% (95\% IC: 88,5-93,5\%) (figuras 1 y 2). Adicionalmente, la gran área observada bajo la curva $\mathrm{ABC}=0,97$ (95\% IC: 0,95-0,98; $\mathrm{P}<0,0001)$ indica que el instrumento presenta una habilidad discriminatoria excelente. De esta forma, al considerarse el estudio de la curva ROC, la administración de la analgesia de rescate debe recomendarse con una puntuación igual o mayor a 8 (26,6\% de la puntuación total).

Cuadro 3. Medianas y semirrango de las puntuaciones de dolor determinadas por el "patrón de referencia" y observadores "ciegos" con el español como lengua materna, a partir del análisis de videos en el período perioperatorio de gatas sometidas a ovariohisterectomía. Medians and semi-range of pain scores determined by blinded observers with Spanish as the first language and 'gold-standard' based on analysis of videos recorded during the perioperative period in cats undergoing ovariohysterectomy.

\begin{tabular}{|c|c|c|c|c|c|c|c|c|}
\hline \multirow{2}{*}{$\begin{array}{l}\text { Momento de } \\
\text { evaluación }\end{array}$} & \multirow{2}{*}{$\begin{array}{l}\text { Puntuaciones de } \\
\text { dolor }\end{array}$} & \multirow{2}{*}{$\begin{array}{l}\text { "Patrón de } \\
\text { referencia" }\end{array}$} & \multicolumn{6}{|c|}{$\begin{array}{c}\text { Observadores "ciegos" con español como lengua materna } \\
\text { Anestesiólogos }\end{array}$} \\
\hline & & & 1 & 2 & 3 & 4 & 5 & 6 \\
\hline \multicolumn{9}{|l|}{ M1 Preoperatorio } \\
\hline & Total $(0-30)$ & $0,0 \pm 2,5$ & $1,0 \pm 5,5$ & $1,5 \pm 5,0$ & $1,0 \pm 3,0$ & $0,0 \pm 1,5$ & $0,0 \pm 4,5$ & $0,0 \pm 3,0$ \\
\hline & Subescala $1(0-15)$ & $0,0 \pm 2,5$ & $1,0 \pm 5,0$ & $1,0 \pm 4,0$ & $0,0 \pm 3,0$ & $0,0 \pm 1,5$ & $0,0 \pm 3,5$ & $0,0 \pm 3,0$ \\
\hline & Subescala $2(0-9)$ & $0,0 \pm 0,0$ & $0,0 \pm 0,5$ & $0,0 \pm 1,0$ & $0,0 \pm 1,0$ & $0,0 \pm 0,5$ & $0,0 \pm 0,0$ & $0,0 \pm 0,0$ \\
\hline & Subescala $3(0-6)$ & $0,0 \pm 0,0$ & $0,0 \pm 0,5$ & $0,0 \pm 1,0$ & $0,0 \pm 0,5$ & $0,0 \pm 0,0$ & $0,0 \pm 1,0$ & $0,0 \pm 0,0$ \\
\hline \multicolumn{9}{|c|}{ M2 Postoperatorio } \\
\hline \multirow{4}{*}{$\begin{array}{l}\text { antes de la } \\
\text { analgesia de } \\
\text { rescate }\end{array}$} & Total (0-30) & $20,5 \pm 7,0 *$ & $22,0 \pm 7,5^{*}$ & $19,5 \pm 8,0^{*}$ & $16,5 \pm 9,5^{*}$ & $17,0 \pm 10,0^{*}$ & $19,0 \pm 10,0^{*}$ & $18,0 \pm 8,5^{*}$ \\
\hline & Subescala $1(0-15)$ & $12,0 \pm 3,0^{*}$ & $13,0 \pm 3,5^{*}$ & $12,0 \pm 5,0^{*}$ & $9,0 \pm 5,5^{*}$ & $11,0 \pm 4,5^{*}$ & $12,0 \pm 5,5^{*}$ & $12,0 \pm 3,5^{*}$ \\
\hline & Subescala $2(0-9)$ & $6,0 \pm 4,5^{*}$ & $6,0 \pm 4,0 *$ & $5,5 \pm 4,5^{*}$ & $5,0 \pm 4,5^{*}$ & $4,0 \pm 4,5^{*}$ & $4,0 \pm 4,5^{*}$ & $5,0 \pm 4,5^{*}$ \\
\hline & Subescala $3(0-6)$ & $3,0 \pm 3,0^{*}$ & $3,0 \pm 3,0^{*}$ & $3,0 \pm 3,0^{*}$ & $2,0 \pm 3,0^{*}$ & $2,0 \pm 3,0^{*}$ & $3,0 \pm 3,0^{*}$ & $3,0 \pm 3,0^{*}$ \\
\hline \multicolumn{9}{|c|}{ M3 Postoperatorio } \\
\hline \multirow{3}{*}{$\begin{array}{l}\text { después de } \\
\text { administrar la } \\
\text { analgesia de } \\
\text { rescate }\end{array}$} & Subescala $1(0-15)$ & $0,0 \pm 2,5 \dagger$ & $\begin{array}{l}2,0 \pm 4,0 \dagger \\
2,0 \pm 3,0 \dagger\end{array}$ & $\begin{array}{l}1,0 \pm 4,0 \dagger \\
1,0 \pm 3,5 \dagger\end{array}$ & $\begin{array}{l}1,0 \pm 2, J \mid \\
0,5 \pm 2,0 \dagger\end{array}$ & $\begin{array}{l}0,0 \pm 2, J \mid \\
0,0 \pm 2,5 \dagger\end{array}$ & $0,0 \pm 2,5 \dagger$ & $0,0 \pm 1,0 \dagger$ \\
\hline & Subescala $2(0-9)$ & $0,0 \pm 0,5 \dagger$ & $0,0 \pm 1,5 \dagger$ & $0,0 \pm 1,0 \dagger$ & $0,0 \pm 1,5 \dagger$ & $0,0 \pm 1,0 \dagger$ & $0,0 \pm 0,0 \dagger$ & $0,0 \pm 0,5 \dagger$ \\
\hline & Subescala $3(0-6)$ & $0,0 \pm 1,0 \dagger$ & $0,0 \pm 1,5 \dagger$ & $0,0 \pm 1,0 \dagger$ & $0,0 \pm 1,0 \dagger$ & $0,0 \pm 1,0 \dagger$ & $0,0 \pm 1,0 \dagger$ & $0,0 \pm 1,0 \dagger$ \\
\hline \multicolumn{9}{|c|}{ M4 Postoperatorio } \\
\hline \multirow{4}{*}{$\begin{array}{l}\text { 24hs después de la } \\
\text { cirugía }\end{array}$} & Total $(0-30)$ & $4,0 \pm 7,0 \dagger$ & $5,0 \pm 8,5 \dagger$ & $5,0 \pm 6,5 \dagger$ & $4,0 \pm 5,0 \dagger$ & $2,0 \pm 4,5 \dagger$ & $3,0 \pm 5,0 \dagger$ & $2,0 \pm 6,0 \dagger$ \\
\hline & Subescala $1(0-15)$ & $0,0 \pm 3,5 \dagger$ & $3,0 \pm 5,0 \dagger$ & $1,0 \pm 4,0 \dagger$ & $0,0 \pm 3,0 \dagger$ & $0,0 \pm 3,0 \dagger$ & $0,0 \pm 3,0 \dagger$ & $0,0 \pm 3,5 \dagger$ \\
\hline & Subescala $2(0-9)$ & $2,0 \pm 3,0 \dagger$ & $2,0 \pm 3,0 \dagger$ & $2,0 \pm 3,0 \dagger$ & $2,0 \pm 4,0 \dagger$ & $1,5 \pm 3,0 \dagger$ & $1,0 \pm 2,5 \dagger$ & $1,0 \pm 3,5 \dagger$ \\
\hline & Subescala $3(0-6)$ & $0,0 \pm 1,0 \dagger$ & $0,0 \pm 1,5 \dagger$ & $0,0 \pm 1,0 \dagger$ & $0,0 \pm 1,0 \dagger$ & $0,0 \pm 1,0 \dagger$ & $0,0 \pm 1,5 \dagger$ & $0,0 \pm 1,0 \dagger$ \\
\hline
\end{tabular}

* Indica validez del contenido: Puntuaciones de dolor en el M2 significativamente mayores que en el M1 (P<0,001).

$\dagger$ Indica validez del constructo: Puntuaciones de dolor en el M3 y M4 significativamente menores que en el M2 (P< 0,001$)$.

Subescala 1 "Alteración psicomotriz" - postura, comodidad, actividad, actitud y miscelánea de comportamientos.

Subescala 2 "Protección del área dolorida y expresión vocal del dolor" - reacción a la palpación de la herida quirúrgica, reacción a la palpación del abdomen/flanco y vocalización.

Subescala 3 "Variables fisiológicas"- Presión arterial y apetito. 


\section{DISCUSIÓN}

El presente estudio apunta a la validación de la versión en español de la escala multidimensional de la UNESPBotucatu para evaluar del dolor postoperatorio en gatos.

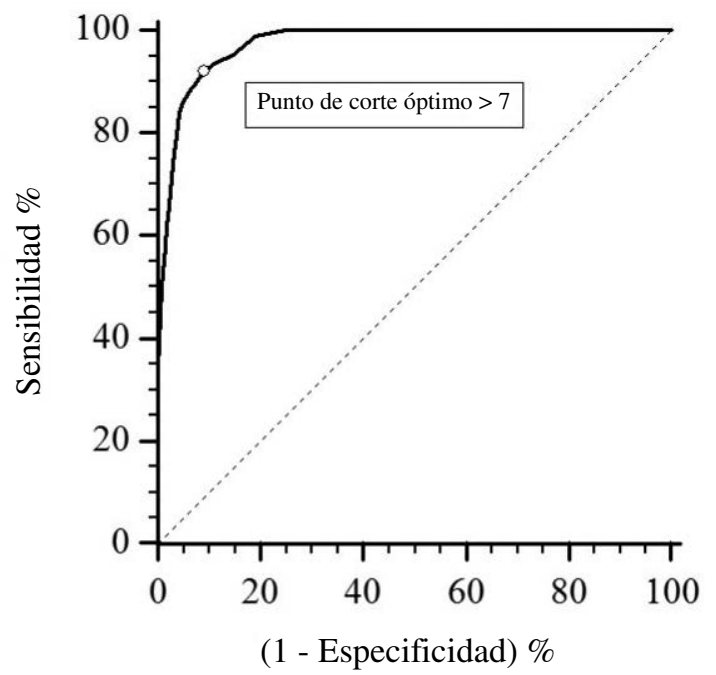

Figura 1. Curva ROC Receiver Operating Characteristic y punto de corte óptimo $>7$, con una sensibilidad del 91,9\%, especificidad de $91,2 \%$ y área bajo la curva de 0,97 , para la versión en español de la escala multidimensional de la UNESP-Botucatu para evaluar el dolor postoperatorio en gatos.

ROC Receiver Operating Characteristic curve and optimal cut-off point $>7$, with sensitivity of $91.9 \%$, specificity of $91.2 \%$, and area under curve of 0.97, of the Spanish version of the UNESP-Botucatu multidimensional composite pain scale to assess postoperative pain in cats.
El proceso de traducción y adaptación cultural y los test psicométricos siguieron las normas estandarizadas de la literatura. Los resultados afianzaron la estructura multidimensional de la escala en la lengua española y comprobaron su validez, confiabilidad, sensibilidad al cambio e interpretabilidad (definición de la puntuación para una intervención analgésica), para evaluar el dolor en gatas sometidas a ovariohisterectomía, al ser utilizada por anestesiólogos de diferentes culturas y con el español como lengua materna.

La multidimensionalidad observada en la escala original en portugués se afianzó en su versión en español. Sin embargo, se detectó una pequeña diferencia relacionada con la estructura factorial. La escala en portugués está formada por cuatro dimensiones o subescalas, ya que el ítem vocalización representa una subescala separada, denominada "expresión vocal del dolor" (Brondani y col 2011). El análisis factorial de la versión en español identificó solo tres dimensiones, ya que el ítem vocalización se incluyó conjuntamente con los ítems reacción a la palpación de la herida quirúrgica y reacción a la palpación del abdomen/ flanco, constituyendo la subescala "protección del área dolorida y expresión vocal del dolor". De esta forma, la estructura multidimensional de la versión en español se mostró superior a la escala original, ya que se considera deseable la presencia de factores con más de dos ítems (Wiseman-Orr y col 2006).

En relación con la consistencia interna de la escala en la versión en español, se han observado excelentes resultados en la puntuación total y en la puntuación parcial de las subescalas "alteración psicomotriz" y "protección del área dolorida y expresión vocal del dolor". Esto permite

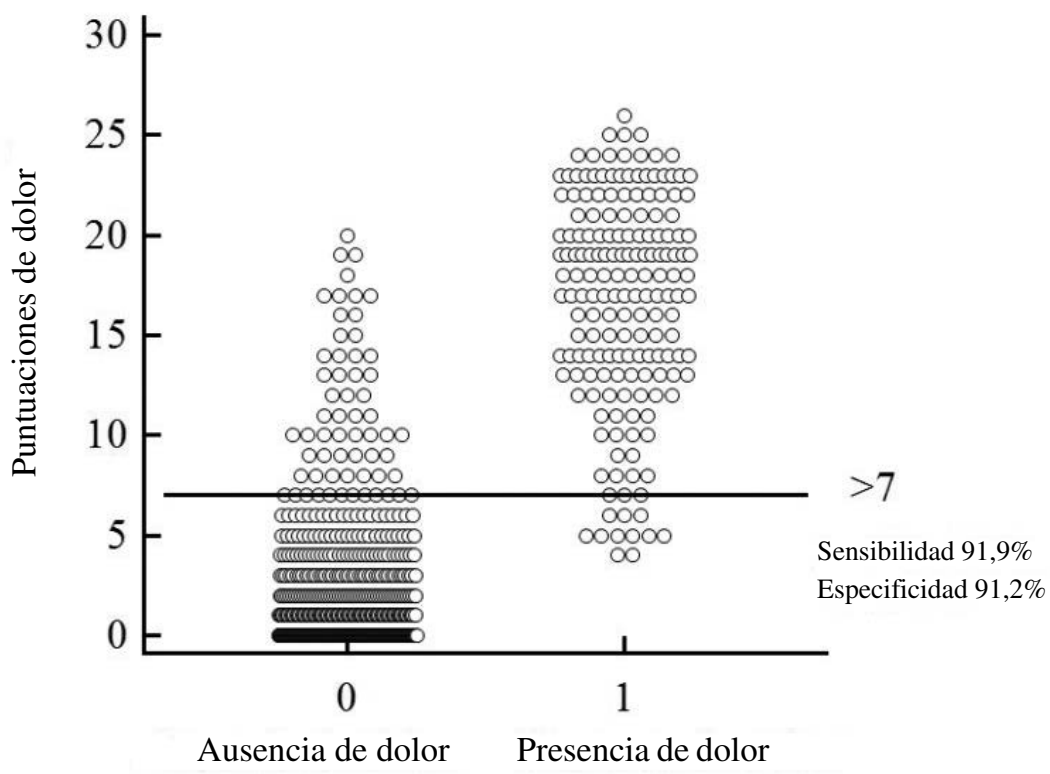

Figura 2. Diagrama que ilustra el punto de corte óptimo para la intervención analgésica de la versión en español de la escala multidimensional de la UNESP-Botucatu para evaluación del dolor postoperatorio en gatos.

Diagram illustrating the optimal cut-off point for rescue analgesia of the Spanish version of the UNESP-Botucatu multidimensional composite pain scale to assess postoperative pain in cats. 
que los resultados obtenidos con el uso de la escala puedan interpretarse a partir de la puntuación total (evaluación global de la intensidad del dolor) o de la puntuación parcial de cada una de las subescalas antes citadas. Por otro lado, la subescala "variables fisiológicas" presentó una consistencia interna moderada $(0,55)$, difiriendo tanto de lo observado en la escala en portugués $(0,76)$ (Brondani y col 2011), como de la versión en inglés $(0,28)$ (Brondani y col $2013^{\text {b }}$ ). La variabilidad observada en la consistencia interna de esta subescala en las diferentes lenguas refleja la propia limitación de los ítems que la componen. Aunque la presión arterial sea el parámetro objetivo que mejor se correlaciona con la presencia de dolor en los gatos (Smith y col 1996), está muy influenciada por otros factores, como por ejemplo el protocolo anestésico-analgésico utilizado. Por ello, la subescala "variables fisiológicas" siempre debe evaluarse asociada a otras subescalas, debiendo evitarse el uso aislado de su puntuación parcial.

La validez del criterio normalmente se evalúa con la correlación de los resultados obtenidos mediante la escala propuesta para validar con otro instrumento, considerado el patrón de referencia (Morton y col 2005). Debido a la inexistencia de una escala patrón de referencia para evaluar el dolor en gatos, la validez del criterio se determinó a partir de la comparación de las puntuaciones de dolor registradas por los evaluadores "ciegos" con el español como lengua materna, con las obtenidas por el evaluador que desarrolló la escala y que se consideró el patrón de referencia. Este método alternativo es similar al utilizado por Gauvain-Piquard y col (1999) durante la validación de una escala para evaluar el dolor en niños. La alta concordancia observada reafirma la validez de criterio de la versión en español, al ser utilizada por anestesiólogos. Es importante caracterizar al grupo de individuos para el que se valida el instrumento (Streiner y Norman 2008).

Las hipótesis propuestas para la validación del contenido y el constructo de la escala, es decir, el aumento de las puntuaciones de dolor tras el procedimiento quirúrgico, y la disminución de las mismas tras administrar la analgesia, respectivamente, se han utilizado ampliamente en la validación de escalas en pediatría (Bullock y Tenenbein 2002, Manworren y Hynan 2003). Este método también evalúa lo responsivo del instrumento (Baeyer y Spagrud 2007). Asimismo, la sensibilidad al cambio se evaluó a partir de la alteración en las puntuaciones de dolor durante el período postoperatorio, abordaje descrito anteriormente en los estudios de validación de escalas de dolor en perros (Morton y col 2005, Murrell y col 2008).

En este estudio, la puntuación total y parcial de las tres subescalas se alteró significativamente en respuesta a la cirugía, a la administración de analgésicos y a lo largo del periodo postoperatorio, lo que confirmó la validez del contenido, el constructo y la sensibilidad al cambio de la escala en la versión en español. Este resultado coincide con las observaciones hechas durante el proceso de validación de la escala original en portugués, así como de su versión en inglés (Brondani y col 2012, 2013a,b). La validación de la puntuación parcial de las subescalas indica que cada aspecto del constructo también puede evaluarse por separado si se desea una evaluación minuciosa, como en las investigaciones científicas. Por otra parte, permite que, de existir alguna dificultad técnica para la evaluación de los ítems de una subescala determinada (por ejemplo, la falta de un equipamiento para medir la presión arterial), esa subescala pueda ser omitida sin comprometer la evaluación global del dolor.

La determinación de la confiabilidad de una escala de evaluación en términos de reproducibilidad y estabilidad, es un requisito fundamental cuando los datos se toman mediante la observación (Beyer y Wells 1989). De esta forma, al considerar la naturaleza observacional de la evaluación del dolor en los animales (Anil y col 2002), este estudio evaluó ambas: la confiabilidad inter e intraobservadores. En su versión en español, todos los ítems de la escala presentaron una confiabilidad adecuada, ya que el nivel de concordancia de las evaluaciones de los diferentes observadores, o del mismo observador en diferentes momentos, varió entre buena y muy buena a partir del análisis del coeficiente de correlación intraclase. Este coeficiente considera el método estadístico más apropiado para el análisis de confiabilidad, aunque presenta limitaciones si la muestra es homogénea (Deyo y col 1991). En nuestro estudio, para garantizar la heterogeneidad de los datos, los análisis se realizaron agrupando los cuatro momentos de evaluación. La confiabilidad de la escala en la versión en español es satisfactoria, y coincide con los resultados observados con la escala original en portugués y su versión en inglés (Brondani y col 2013 $3^{\mathrm{a}, \mathrm{b}}$ ). Podemos atribuir la reproducibilidad y la estabilidad de la escala a la descripción detallada de los comportamientos de dolor que el investigador debe observar, lo que consecuentemente reduce la subjetividad del análisis.

La disponibilidad de una puntuación de intervención analgésica es un requisito esencial en una escala de evaluación de dolor, ya que ayuda al clínico a decidir el tratamiento analgésico (Reid y col 2007). El presente estudio utilizó el análisis de la curva ROC para determinar la puntuación mínima relacionada con la necesidad de intervención analgésica, la misma metodología aplicada en la escala original y su versión en inglés (Brondani y col $2013^{\mathrm{a}, \mathrm{b}}$ ). Aunque esta técnica estadística sea innovadora en medicina veterinaria, ya se ha usado en medicina humana (Hünseler y col 2011). Permite determinar la habilidad de un test para discriminar grupos, establecer un punto de corte óptimo y comparar el desempeño de dos o más test (Streiner y Cairney 2007). Igual que en la escala en portugués y en inglés (Brondani y col 2013 $3^{\mathrm{a}, \mathrm{b}}$ ), el punto de corte óptimo identificado para la escala en la versión en español fue $>7$, lo que significa que la terapia analgésica deberá instaurarse en puntuaciones $\geq 8(26,6 \%$ de la puntuación total). Sin embargo, no se debe negar el uso de analgésicos en animales con puntuación $\leq 7$ si el juicio clínico del profesional indica que deben administrarse. 
Tras considerar los resultados que acabamos de presentar, podemos concluir que la versión en español de la escala multidimensional de la UNESP-Botucatu es un instrumento interpretable (presenta una puntuación para la intervención analgésica), válido y confiable para la evaluación del dolor agudo en gatas sometidas a ovariohisterectomía, al ser utilizado por anestesiólogos con el español como lengua materna.

\section{AGRADECIMIENTOS}

Los autores agradecen al Consejo Nacional de Desarrollo Científico y Tecnológico (CNPq-Brasil) por el apoyo financiero, y al Dr. Juan CD Moreno y Dr. Jean GF Joaquim por la colaboración prestada durante el proceso de traducción/adaptación cultural de la escala al español.

\section{REFERENCIAS}

Altman DG. 1991. Some common problems in medical research. In Altman DG (ed). Practical statistics for medical research. Chapman and Hall, London, UK, Pp 404-408.

Anil SS, I Anil, J Deen. 2002. Challenges of pain assessment in domestic animals. J Am Vet Med Assoc 220, 313-319.

Baeyer VC, LJ Spagrud. 2007. Systematic review of observational (behavioral) measures of pain for children and adolescents aged 3 to 18 years. Pain 127, 140-150.

Beaton DE, C Bombardier, F Guillemin, MB Ferraz. 2000. Guidelines for the process of cross-cultural adaptation of self-report measures. Spine 25, 3186-3191.

Beyer JE, N Wells. 1989. The assessment of pain in children. Pediatr Clin North America 36, 837-854.

Brondani JT, SPL Luna, CR Padovani. 2011. Refinement and initial validation of a multidimensional composite scale for use in assessing acute postoperative pain in cats. Am J Vet Res 72, 174-183.

Brondani JT, SPL Luna, BW Minto, BPR Santos, SL Beier, LM Matsubara, CR Padovani. 2012. Validade e responsividade de uma escala multidimensional para avaliação de dor pós-operatória em gatos. Arq Bras Med Vet Zootec 64, 1529-1538.

Brondani JT, SPL Luna, BW Minto, BPR Santos, SL Beier, LM Matsubara, CR Padovani. 2013 . Confiabilidade e pontuação minima relacionada à intervenção analgésica de uma escala multidimensional para avaliação de dor pós-operatória em gatos. Arq Bras Med Vet Zootec, 65, 153-162.

Brondani JT, KR Mama, SPL Luna, BD Wright, S Niyom, J Ambrosio, P Vogel. 2013 ${ }^{\mathrm{b}}$. Validation of the English version of the UNESPBotucatu multidimensional composite pain scale for assessing postoperative pain in cats. BMC Vet Res 9, 143.

Bullock B, M Tenenbein. 2002. Validation of 2 pain scales for use in the pediatric emergency department. Pediatrics 110, 1-6.
Deyo RA, P Diehr, DL Patrick. 1991. Reproducibility and responsiveness of a health status measures. Control Clin Trials 12, 142-158.

Gauvain-Piquard A, C Rodary, A Rezvani, S Serbouti. 1999. The development of the $\mathrm{DEGR}^{\mathrm{R}}$ : a scale to assess pain in young children with cancer. Eur J Pain 3, 165-176.

Guillemin F, C Bombardier, D Beaton. 1993. Cross-cultural adaptation of health-related quality of life measures: literature review and proposed guidelines. J Clin Epidemiol 46, 1417-1432.

Holton LL, EM Scott, AM Nolan, J Reid, E Welsh, D Flaherty. 1998. Comparison of three methods used for assessment o pain in dogs. J Am Vet Med Assoc 212, 61-66.

Holton L, J Reid, M Scott, P Pawson, A Nolan. 2001. Development of a behavior-based scale to measure acute pain in dogs. Vet Rec $148,525-531$

Hünseler C, V Merkt, M Gerloff, F Eifinger, A Kribs, B Roth. 2011. Assessing pain in ventilated newborns and infants: validation of the Hartwig score. Eur J Pediatr 170, 837-853.

Jensen MP. 2003. Questionnaire validation: a brief guide for readers of the research literature. Clin J Pain 19, 345-352.

Kaiser HF. 1958. The varimax criterion for analytic rotation in factor analysis. Psychometrika 23, 187-200.

Manworren RC, L Hynan. 2003. Clinical validation of FLACC: preverbal patient pain scale. Pediatr Nurs 29, 140-146.

Morton CM, J Reid, ME Scott, LL Holton, AM Nolan. 2005. Application of a scaling model to establish and validate an interval level pain scale for assessment of acute pain in dogs. Am J Vet Res 66, 2154-2166.

Murrell JC, EP Psatha, EM Scott, J Reid, LJ Hellebrekers. 2008. Application of a modified form of the Glasgow pain scale in a veterinary teaching centre in the Netherlands. Vet Rec 162, 403-408.

Reid J, AM Nolan, JM Hughes, D Lascelles, P Pawson, EM Scott. 2007. Development of the short-form Glasgow composite measure pain scale (CMPS-SF) and derivation of an analgesic intervention score. Anim Welf 16, 97-104.

Smith JD, SW Allen, JE Quandt, RL Tackett. 1996. Indicators of postoperative pain in cats and correlation with clinical criteria. Am $J$ Vet Res 57, 1674-1678.

Souza FF, Silva JA. 2005. A métrica da dor (dormetria): problemas teóricos e metodológicos. Rev Dor 6, 469-513.

Souza VD, W Rojjanasrirat. 2011. Translation, adaptation and validation of instruments or scales for use in cross-cultural health care research: a clear and user-friendly guideline. J Eval Clin Pract 17, 268-274.

Streiner DL, J Cairney. 2007. What's under the ROC? An introduction to receiver operating characteristics curves. Can J Psychiatry 52, 121-128.

Streiner DL, GR Norman. 2008. Health measurement scales: a practical guide to their development and use. $4^{\text {th }}$ ed. Oxford University Press, New York, USA.

Wiseman-Orr ML, EM Scott, J Reid, AM Nolan. 2006. Validation of a structured questionnaire as an instrument of measure chronic pain in dogs on the basis of effects on health-related quality of life. Am $J$ Vet Res 67, 1826-1836. 